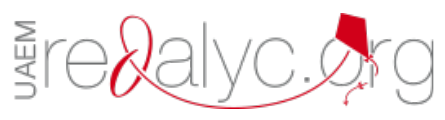

Centro Sur

ISSN: $2600-5743$

compasacademico@icloud.com

Grupo Compás

Ecuador

\title{
Tendencias en formación y desarrollo a la gestión del talento por competencias
}

Pazmiño Santos Mae, Wendy

Tendencias en formación y desarrollo a la gestión del talento por competencias

Centro Sur, vol. 2, núm. 1, 2018

Grupo Compás, Ecuador

Disponible en: http://www.redalyc.org/articulo.oa?id=588861692001 


\title{
Tendencias en formación y desarrollo a la gestión del talento por competencias
}

Trends in training and development for talent management by competences

\author{
Wendy Pazmiño Santos Mae wendy.pazminos@ug.edu.ec \\ Universidad de Guayaquil, Ecuador \\ http://orcid.org/0000-0003-3355-0378
}

Centro Sur, vol. 2, núm. 1, 2018

Grupo Compás, Ecuador

Recepción: 13 Junio 2017 Aprobación: 24 Julio 2018

Redalyc: http://www.redalyc.org/ articulo.oa?id $=588861692001$
Resumen: Decidir qué carrera se quiere estudiar no siempre es tarea fácil, ya que se está definiendo su futuro y a lo que se dedicará el resto de su vida. La planificación de la plantilla se está convirtiendo en un factor clave para determinar las necesidades de talento futuras y hay un énfasis creciente en el desarrollo de procesos de evaluación del desempeño. Hemos de pasar de concebir el mundo laboral desde un lugar de esfuerzo a un espacio en el que utilicemos el juego y el desafío para aprender. Cuando se aplican estas técnicas, se logra alcanzar resultados, mejora el compromiso y se consiguen espacios más creativos e innovadores. Los acelerados de cambios en el mundo profesional y la creciente globalización están obligando a redefinir el desarrollo de carrera asegurado que el empleo para toda la vida ha muerto y que tenemos que prepararnos para pilotar nuestra propia carrera, senderos que nunca serán rectos sino helicoidales, con etapas en las que el movimiento será transversal y etapas en las habrá rotación y cambio pero no aumento salarial sino un equilibrio dinámico que representa una mayor complejidad y variabilidad.

Palabras clave: Complejidad y Variabilidad, Evaluación, Desempeño Laboral, Planificación, Profesional.

Abstract: Deciding what career you want to study is not always easy, because you are defining your future and what the rest of his life will be dedicated. The workforce planning is becoming a key factor in determining the needs of future talent and there is a growing emphasis on the development of performance evaluation processes. We must move to conceive the world of work from a place of effort within a space in which we use the game and the challenge to learn. When these techniques are applied, it is possible to achieve results, improving engagement and more creative and innovative spaces are achieved. The rapid changes in the professional world and increasing globalization are forcing redefine career development ensured that employment for life is dead and that we must prepare to pilot our own career paths that will never be straight but spiral, with stages in which the movement will cross and stages in the rotation and will change but no wage increase but a dynamic balance that represents more complexity and variability. Keywords: Complexity and Variability, Evaluation Planning, Professional, Work Performance.

\section{INTRODUCCIÓN}

La importancia del liderazgo en los procesos de cambio. "Todo cambio lleva implícitos elementos de renuncia y pérdida, pero también elementos de aprendizaje y mejora. Sólo aquellas organizaciones que decidan conscientemente emprender un proceso de cambio serán las que puedan obtener resultados positivos", ha apuntado Langa. "El cambio -ha continuado- debe diseñarse, debe liderarse y debe implicar e ilusionar a toda la organización." 
La gestión de carrera y sucesión está viviendo un cambio de gran envergadura. De ser un proceso focalizado en el relevo en las posiciones clave de las compañías, está evolucionando para centrar los esfuerzos en la retención, desarrollo y compromiso de los empleados en todos los niveles de las organizaciones.

El aprendizaje constante es esencial para nuestro éxito, así como para tener los mejores talentos de la industria. Por eso generamos conocimiento a partir de las habilidades básicas para una industria específica y lo ponemos a disposición de todos.

El fortalecimiento de tus habilidades y conocimiento, es sólo una parte de la construcción de un mejor Performance Achivement; que se centra en los empleados individualmente: sus fortalezas, su progreso y sus posibilidades de carrera.

En este nuevo mundo laboral las barreras entre el trabajo y la vida persisten. En la actualidad se han encontrado graduados, egresados y profesionales de diferentes áreas o ramas desmotivados porque en sus empresas no les proporcionaban formación para desarrollarse y mejorar su trayectoria profesional. He coincidido con profesionales que cambiaban de empresa y proyecto por el único motivo de recibir más formación en su nueva organización. La posibilidad de formación y desarrollo profesional es (¿era?) uno de los factores de motivación más determinantes a la hora de seleccionar un proyecto profesional.

Hoy en día se puede entender la situación que vive el profesional, pero lo que nunca se ha logrado entender es que algunos profesionales hagan responsables de su formación académica y docentes y desarrollo a sus empresas y a sus jefes, que sitúen el centro de responsabilidad de su carrera profesional en otras personas, ya que considero que los principales responsables de nuestro desarrollo profesional somos nosotros mismos, ya que el desarrollo es una parte importante de nuestras vidas y es continuo.

Por suerte esto empieza a pertenecer al pasado, en estos nuevos tiempos de "sociedad red" tenemos tantas herramientas para acceder al aprendizaje continuo que de nada sirven las auto-escusas. Las organizaciones deben permitir y pueden facilitar nuestro aprendizaje, pero somos nosotros los que tenemos que tomar la iniciativa y asumir la responsabilidad de nuestro propio futuro.

Los programas de formación se adaptan a las necesidades individuales y los cursos que los empleados eligen dependen de sus habilidades, experiencias y áreas de interés. Nuestro portal global ofrece más de 50.000 cursos online, aulas virtuales y otros recursos clave de aprendizaje. Nuestros profesionales pueden encontrar recursos para adquirir nuevas habilidades y mejorar las actuales. Además, proporcionamos a los empleados programas de formación, calendario de los cursos y los enlaces para su registro.

Por supuesto, y dado que el desarrollo profesional se encuentra en el centro de todo lo que hacemos, continuaremos buscando nuevas formas de aprender e incorporar ideas innovadoras. Así, al trabajar con empresas líderes a nivel mundial, aprenderás también en el día a día de trabajo, 
interactuando con clientes, miembros del equipo del proyecto, mentores y otros profesionales.

Conceptos como el de sociedad red han revolucionado por completo el panorama de la formación en el desempeño y desarrollo de las carreras profesionales.

Son muchos los estudiosos del tema que consideran que se deben superar el tópico de que una empresa buena es la que dedica tiempo a la formación de sus trabajadores, porque las posibilidades del empleado para completarlas superan ya el ámbito de la empresa e impregnan la vida diaria del trabajador conectado a la red.

Actualmente se está desarrollando dentro de la sociedad del conocimiento y disponemos de las herramientas necesarias para que nos hagamos responsables de nuestra formación y desarrollo profesional. De ahí se extraerá cuán buenos somos para mantener esa formación de manera constante a lo largo de nuestra vida laboral.

La empresa responsable será aquella que permita el desarrollo profesional para las carrera acorde a las necesidades laborales, pero eres tú el que debes asumir la responsabilidad de estar siempre atento a las nuevas posibilidades que existen para poder formarte

Actualmente, los expertos en temas de formación comparten la necesidad de establecer un Entorno Personal de Aprendizaje (PLE son sus siglas en inglés). Esta será una labor que el empleado, orientado y apoyado por la empresa, realizará para organizar:

$\varnothing$ El conjunto de herramientas

$\varnothing$ Las fuentes de información

$\varnothing \mathrm{Y}$ las conexiones y actividades que cada persona emplea para mantener su formación constante.

Este proceso de aprendizaje se estructura mediante la formación en la red y atendiendo a los objetivos que decida cada usuario. Carece de estructura formal, no responde a títulos académicos, ni se evalúan los resultados. Se trata de recursos que no suponen costes para los beneficiarios. Son dos los recursos con los que cuentas para desarrollar tu entorno de aprendizaje:

Comunidades de práctica virtuales. En la que se produce la interacción virtual constante entre un grupo de personas que comparten preocupaciones, intereses comunes y conocimientos.

La tarea del formador o mediador es fundamental, porque establece y allana el camino para poder adquirir las herramientas y competencias de este nuevo tipo de aprendizaje.

La gestión de carrera y sucesión se ha convertido en un proceso consolidado de Recursos Humanos, inicialmente en las grandes necesidades de las empresas, pero más recientemente también en las pequeñas organizaciones. El énfasis se continúa situando en las posiciones críticas en los niveles ejecutivos, pero los procesos ya no son sólo para esos roles. Se utilizan cada vez más para puestos de trabajo especializados y los que no son de liderazgo.

Además, la gestión de carrera y sucesión es clave para mejorar el compromiso de los millenials, que requieren de una mayor transparencia 
sobre las oportunidades disponibles y del apoyo de los managers en sus próximos pasos de carrera.

Las áreas de Formación y Desarrollo tienen ante sí la compleja y a la vez atractiva tarea de acompañar al negocio proporcionándole herramientas de recualificación que permitan adaptar las competencias del empleado a la rápida evolución del mercado.

Quizá éste sea uno de los momentos más complicados para los profesionales en la gestión de personas, porque el mundo empresarial y el mercado laboral están en constante cambio, porque los departamentos de recursos humanos de hoy no se parecerán a los que funcionen dentro de cinco años, y porque lo más probable es que en dos décadas se habrán creado profesiones y formas de trabajar que ni siquiera somos capaces de imaginar.

El cambio vertiginoso que imponen mercados, cada vez más globales, competitivos y complejos a las organizaciones, necesariamente impacta en su desempeño esperado, no sólo en términos de supervivencia, sino también de competitividad. Como es de suponer, ello exige a las empresas significativas reestructuraciones internas, a la que no escapan las estructuras organizativas y por ende la Gestión del Talento Humano.

En una organización del trabajo de conformación más horizontal y que prioriza el valor estratégico del conocimiento, las relaciones jerárquicas tienden a ser sustituidas por grupos de trabajos con mayor responsabilidad y poder. Parece evidente que las organizaciones del futuro facilitarán mayor y mejor información, así como también se apoyarán más en las personas y menos en los puestos de trabajo.

Por lo pronto, las decisiones estratégicas que se puedan adoptar desde una empresa en tal sentido, necesariamente están vinculadas con la reestructura de sus programas de Gestión del Talento Humano. Es en este contexto que el modelo de competencias aparece como una nueva modalidad de gestión, cuyo principal objetivo es asegurar que las personas asignadas a las distintas actividades sean las más idóneas para la función. A su vez, permite integrar en torno al concepto de competencias todos los subsistemas que conforman la Gestión del Talento Humano (selección, inducción, planes de carrera, capacitación, evaluación del desempeño, desvinculación, etc).

\section{MATERIALES Y MÉTODOS}

El concepto de competencias no es nuevo, pero la gestión por competencias crece en importancia en el mundo empresarial: su aplicación ofrece la novedad de un estilo de dirección en el que prima el factor humano, en el que cada persona, empezando por los propios directivos, deben aportar sus mejores cualidades profesionales y personales a la organización. Lo oportuno de este enfoque es que su concepción básica reconoce que el Talento Humano de la empresa es el que le permite lograr una ventaja competitiva sostenible en el largo plazo.

Con el término gestión hacemos referencia aquí al conjunto de acciones didáctico organizativa necesaria para lograr una meta final determinada 
centrada en la construcción, transferencia y adquisición de conocimiento en la totalidad de las áreas de formación. El talento humano hay que entenderlo como la "capacidad para obtener resultados notables con el ejercicio de la inteligencia” (Moliner, M. 2004). En nuestro caso, dicha actividad es el adecuado desarrollo de las capacidades para la enseñanza (profesores) y el aprendizaje (estudiantes). Las acción gestora aquí estará fundamentada en una serie de decisiones en el ámbito de la antropología, lo disciplinar y la metodología didáctica.

\section{RESULTADOS}

Es importante recordar que llevar a cabo esta práctica implica también una visión y colaboración de la dirección general para estructurar y guiar los equipos de trabajo, los procesos y las herramientas tecnológicas orientado estos recursos a la consecución de las metas empresariales globales.

Las competencias son un enfoque para la educación y no un modelo pedagógico, pues no pretenden ser una representación ideal de todo el proceso educativo, determinando cómo debe ser el proceso instructivo, el proceso desarrollador, la concepción curricular, la concepción didáctica y el tipo de estrategias didácticas a implementar.

En este sentido, (Tobón, 2007) como bien se expone, "el enfoque de competencias puede llevarse a cabo desde cualquiera de los modelos pedagógicos existentes, o también desde una integración de ellos". Pag. 17.

"Es una característica de personalidad, devenidas de comportamientos, que generan un desempeño exitoso en un puesto trabajo."11 Todas las personas tienen un conjunto de atributos y conocimientos adquiridos o innatos que definen sus competencias para una cierta actividad. Sin embargo para descubrir las competencias no se necesita estudiar el perfil físico, psicológico o emocional, solamente interesan aquellas características que hagan eficaces a las personas dentro de la organización. (ALLES, Martha, 2008)

En la nueva tendencia de gestión de Talento Humano frente a competencias de carreras del mañana, el desarrollo profesional del talento es sin duda, una ventaja competitiva sobre las empresas. Sobre esto, hoy te presentamos dos puntos de vista del por qué el desarrollo profesional debe ser una prioridad en tu compañía

En la actualidad hay que olvidarse de carreras de derecho y la administración de empresas. Desarrolladores de software, ingenieros biomédicos o analista de datos son algunas de las carreras de la era actual, que a mediano plazo comienzan a perfilarse como las más demandadas en el mundo.

En base a los nuevos cambios que solicita el Consejo de Educación Superior "CES" en base a su Reglamento de Régimen Académico los profesionales de Genética, Ingeniería Ambiental, Psicología y Salud Mental, Ciencia de los alimentos, Ingeniería de software, Seguridad informática, Medicina, Ingeniería informática e Ingeniería Civil serán requeridos en todos los países del mundo, se destaca en un artículo publicado en el sitio Ehowenespanol.com. 
Tomando como base la sociedad actual y en base a algunas proyecciones, es posible aventurar cuáles serán las carreras del futuro, afirma la publicación.

\section{DISCUSIÓN}

Implementar en la empresa este enfoque de gestión del talento humano por competencias se vuelve una tarea más sencilla y eficiente cuando se administra mediante una solución tecnológica como SuccessFactors que integra los diversos procesos referentes a tu capital humano ya que esto te permitirá tener una visión integral logrando el objetivo final que es contar con un equipo de trabajo desarrollado, preparado y potencializado, el cual estará motivado y comprometido a formar una sinergia con la empresa para alcanzar las metas y objetivos.

A través de una política de recursos humanos se busca la formación de personas que vayan creciendo profesional y personalmente dentro de la organización. Siguiendo el enfoque de competencias, el plan de carreras es un método que determina las tareas organizativas y los conocimientos y habilidades clave a desarrollar para un desempeño superior, ya sea en el puesto de trabajo actual o en otro de la organización. Con el canal de información que proporcionan las competencias es posible identificar las características y aptitudes de cada persona y de cada puesto para proponer un plan de carrera adaptado a ellas.

Es fundamental entonces transformar nuestra visión, desde la economía a la economía humana, desde el Capital de Trabajo hacia el desarrollo, potencialización del Capital Humano, que es lo que en esta década le dará la única ventaja competitiva sustentable en el tiempo a la empresa.

Por otro lado, aunque el Sistema de Certificación de Competencias sea difícil de desarrollar y de llevar a la realidad, debe ser un esfuerzo continuo y sobre todo compartido por los sectores gubernamentales, privado y educativo, de tal manera que una alianza entre ellos derive en el incremento de la productividad, las ganancias, el empleo y el sistema de capitales basados en conocimientos que se buscan hoy en día.

\section{Referencias}

1. ALLES, M. (2008). Dirección Estratégica de Recursos Humanos, Gestión por competencias. Argentina: Ediciones Gránica.

2. CHANG, R.Y. (1994). Trabajar en equipo para triunfar. Argentina: Ediciones Granica S.A.

3. Universidad Católica del Norte (2005). Talento Humano. Chile:CEDUCN. Recuperado de http://www.ced.ucn.cl/s_taller_2005/temas/ talento_humano.htm.

4. Moliner, M. (2004). Diccionario del uso del español. Tomos A-H e I-Z. . Madrid: Editorial Gredos.

5. Tobón, S. (2007). El enfoque complejo de las competencias y el diseño curricular. Acción pedagógica, 16(1), 14-28. 
Centro Sur, 2018, 2(1), Enero-Junio, ISSN: 2600-5743 